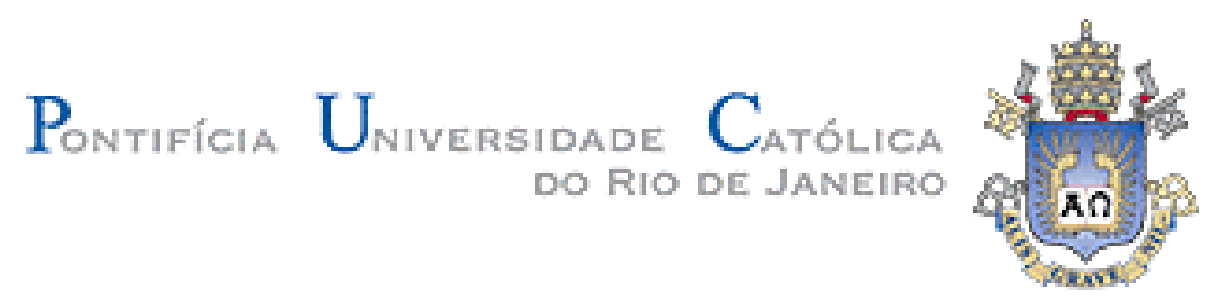

Tania Vanessa Bustamante Ubillús

\title{
Avaliação do Comportamento Dinâmico de um Muro de Gravidade
}

Dissertação de Mestrado

Dissertação apresentada ao Programa de PósGraduação em Engenharia Civil da PUC-Rio como requisito parcial para obtenção do título de Mestre em Engenharia Civil.

Orientador: Prof. Celso Romanel

Rio de Janeiro

Março de 2010 


\section{Pontificia U Uiversidade Católica $_{\text {als }}$

Tania Vanessa Bustamante Ubillús

\section{Avaliação do Comportamento Dinâmico de um Muro de Gravidade}

Dissertação apresentada como requisito parcial para obtenção do grau de Mestre pelo Programa de PósGraduação em Engenharia Civil da PUC-Rio. Aprovada pela Comissão Examinadora abaixo assinada.

Prof. Celso Romanel Orientador Departamento de Engenharia Civil - PUC-Rio

$\mathrm{Dr}^{\mathrm{a}}$. Andréia Abreu Diniz de Almeida Departamento de Engenharia Civil - PUC-Rio

Prof ${ }^{a}$. Bernadete Ragoni Danziger UERJ

Dr. Francisco Claudio Pereira de Barros CNEN

Prof. José Eugênio Leal Coordenador Setorial do CentroTécnico Científico - PUC - Rio

Rio de Janeiro, 05 de março de 2010 
Todos os direitos reservados. É proibida a reprodução total ou parcial do trabalho sem autorização da universidade, da autora e do orientador.

\section{Tania Vanessa Bustamante Ubillús}

Graduou-se em Engenharia Civil pela Universidad Nacional de Ingenieria de Lima - Perú em 2005. Principais áreas de interesse: dinâmica de solos, geomecânica computacional e mineração.

Ficha Catalográfica

Ubillús, Tania Vanessa Bustamante

Avaliação do comportamento dinâmico de um muro de gravidade / Tania Vanessa Bustamante Ubillús; orientador: Celso Romanel. - 2010.

118 f. : il. (color.) ; $30 \mathrm{~cm}$

Dissertação (mestrado)-Pontifícia Universidade Católica do Rio de Janeiro, Departamento de Engenharia Civil, 2010.

Inclui bibliografia

1. Engenharia civil - Teses. 2. Comportamento dinâmico. 3. Estruturas de contenção. 4. Método pseudoestático. 5. Deslocamento permanente. 6. Método dos elementos finitos. 7. Carregamento sísmico. I. Romanel, Celso. II. Pontifícia Universidade Católica do Rio de Janeiro. Departamento de Engenharia Civil. III. Título. 

dedicação. Ao meu querido irmão, com muito amor sempre 


\section{Agradecimentos}

A Deus pela sua benção todos os dias de nossas vidas.

Aos meus pais pelo apoio incondicional nesta importante etapa da minha vida profissional.

Ao meu irmão pelo carinho, por estar sempre perto de mim e acreditar na minha pessoa.

Ao professor Celso Romanel pela dedicada orientação no desenvolvimento deste trabalho e, em especial por sua valiosa amizade.

À Pontifícia Universidade Católica de Rio de Janeiro, por ter me concedido a oportunidade de ser parte desta casa de estudos.

Ao Brasil e ao CNPq pelo apoio financeiro.

À minha Tati por sua adorável preocupação por mim.

A todos os professores do Departamento da Engenharia Civil, pelos conhecimentos transmitidos em cada uma das disciplinas que cursei.

Aos professores Bernadete, Andréia e Francisco Claudio, membros da minha Comissão Examinadora, pelas sugestões neste trabalho.

Ao Vector Perú S.A.C. pelo grande apoio, ao Denys Parra e ao Mark Smith pela confiança depositada, fico grata a vocês.

Aos meus queridos amigos da PUC-Rio que fizeram do dia a dia um convívio agradável.

Aos funcionários da Secretaria do DEC pela amável atenção. 


\section{Resumo}

Bustamante Ubillús, Tania Vanessa; Romanel, Celso (orientador) Avaliação do Comportamento Dinâmico de um Muro de Gravidade. Rio de Janeiro, 2010. 118 p. Dissertação de Mestrado - Departamento de Engenharia Civil, Pontifícia Universidade Católica do Rio de Janeiro.

O projeto de estruturas de contenção de solos submetidas a carregamentos dinâmicos é um importante tema da engenharia geotécnica, principalmente nos países andinos. A abordagem mais comum consiste no emprego de métodos de equilíbrio limite (pseudo-estáticos) ou de técnicas baseadas na formulação de Newmark (1965) para cálculo de deslocamentos permanentes da estrutura. Um método pseudo-estático clássico é o apresentado por Mononobe-Okabe (1929) como uma extensão da teoria de Coulomb (1773), enquanto que Richards e Elms (1979) sugeriram um método de projeto baseado em deslocamentos permanentes. Nesta dissertação os resultados da aplicação do método dos elementos finitos na análise dinâmica do comportamento de um muro de gravidade são comparados com aqueles previstos por diversos outros métodos analíticos propostos na literatura, tanto em termos do cálculo de empuxos quanto de deslocamentos permanentes. As vantagens e limitações destes métodos são discutidas, bem como são discutidos vários aspectos da modelagem numérica que devem ser cuidadosamente considerados pelo engenheiro geotécnico para obter uma simulação representativa do problema dinâmico.

\section{Palavras - chave}

Comportamento dinâmico; estruturas de contenção; método pseudo-estático; deslocamento permanente; método dos elementos finitos; carregamento sísmico. 


\section{Abstract}

Bustamante Ubillús, Tania Vanessa; Romanel, Celso (advisor). Evaluation of the Dynamic Behavior of a Gravity Wall. Rio de Janeiro, 2010. 118p. M.Sc. Dissertation - Departamento de Engenharia Civil, Pontifícia Universidade Católica do Rio de Janeiro.

The design of earth retaining structures during earthquakes is an important subject of the geotechnical engineering, mainly in the Andesian countries of South America. The most common approach consists in the use of some quasi-static method or technique based on Newmark's (1965) model to estimate inelastic displacements of the structure. The classical quasi-static method is the one proposed by Mononobe -Okabe (1926, 1929), as an extension of the Coulomb's (1773) theory to a dynamic context, while Richards and Elms (1979) suggested a design method based on inelastic displacements. In this thesis the dynamic response of a gravity retaining wall obtained through the finite element method are compared with those predicted by several other analytical formulation proposed in the literature, concerning both the resultant of the earth pressures and the wall displacements experienced during the earthquake. The advantages and shortcomings in the application of all these methods are discussed herein, as well as several modeling aspects are discussed that must be carefully considered by the geotechnical engineer in order to obtain a representative simulation of the dynamical problem.

\section{Keywords}

Dynamic behavior; earth retaining structures; quasi-static method; inelastic displacement; finite element method; seismic load. 


\section{Sumário}

1. Introdução 20

1.1. Motivação e objetivos 20

1.2. Estrutura da dissertação 23

2. Análise do comportamento estático 24

2.1. Introdução 24

2.2. Coeficientes de empuxo 28

2.3. Métodos de equilíbrio limite 32

2.3.1. Método de Rankine (1857) 32

2.3.2. Método de Coulomb (1776) 39

3. Análise do Comportamento Dinâmico 44

3.1. Métodos Pseudo-Estáticos 45

3.1.1. Método Mononobe-Okabe (M-O, 1926) 45

3.1.2. Método de Seed e Withman (1970) 50

3.1.3. Método de Steedman-Zeng (1990) 50

3.2. Métodos Elásticos 53

3.2.1. Método de Wood (1973) 54

3.3. Métodos Elasto-Plásticos 56

3.3.1. Aspectos da modelagem dinâmica $\quad 57$

4. Análise dos Deslocamentos Permanentes 65

4.1. Método de Richards-Elms (1979) 65

4.1.1. Cálculo das forças $\quad 65$

4.1.2. Cálculo dos deslocamentos 71

4.2. Método de Whitman-Liao (1985) 75

5 Análise Numérica $\quad 78$

5.1. O programa computacional PLAXIS 78

$\begin{array}{ll}\text { 5.2. Descrição do problema } & 79\end{array}$ 
5.3. Análise estática $\quad 81$

5.4. Análise Pseudo-Estática 84

5.5. Análise dinâmica 85

5.5.1. Modelo de elementos finitos 85

5.5.2. Análise dos deslocamentos permanentes 87

6. Conclusões e sugestões 103

6.1. Conclusões 103

6.2. Sugestões 105

Referências Bibliográficas 106

APÊNDICE 113

A.1. Método de Newmark (1965) 113 


\section{Lista de figuras}

Figura 1.1 - Tipos comuns de estruturas de contenção de terra (modificado de Kramer, 1996).

Figura 2.1 - Utilização de estruturas de contenção (modificado

de Huntington,1957).

Figura 2.2 - Mecanismos típicos de ruptura para um muro de gravidade:

(a) ruptura por deslizamento (translacional); (b) ruptura por

tombamento (rotacional); (c) ruptura por instabilidade global. Referencia

Kramer - 1996.

Figura 2.3 - Forças atuantes sobre estrutura de contenção (modificado

de Huntington,1957).

Figura 2.4 - Esquema ilustrativo utilizado na definição dos coeficientes de empuxo ativo e passivo (modificado de Perloff \& Baron - 1976).

Figura 2.5 - Tensões horizontais nos pontos A e B do solo - (modificado de Perloff \& Baron - 1976)

Figura 2.6 - Círculos de Mohr correspondentes aos estados de tensão inicial e final para os pontos de solo A (ruptura ativa) e B (ruptura passiva), modificado de Lemos, 1997.

Figura 2.7 - Aplicação do método de Rankine para a obtenção de cunhas de ruptura no solo e cálculo dos valores de empuxo sobre estruturas de contenção (modificado de Perloff \& Baron, 1976).

Figura 2.8 - Formato das superfícies de ruptura obtidas pelo método de Rankine quando se considera o atrito na interface solo - muro (modificado de Perloff \& Baron, 1976).

Figura 2.9 - Distribuição de tensões horizontais na interface solo-estrutura para diversas combinações de solo no estado ativo: a) $\phi \neq 0, \mathrm{c}=0$;

b) $\phi=0, c \neq 0$; c) $\phi \neq 0, c \neq 0$ (NAVFAC ,1982).

Figura 2.10 - Distribuição de tensões horizontais na interface solo-estrutura para diversas combinações de solo no estado passivo: a) $\phi \neq 0, c=0$;

b) $\phi=0, c \neq 0 ; c) \phi \neq 0, c \neq 0$ (NAVFAC ,1982). 
Figura 2.11 - Cunha ativa de solo delimitada pela superfície do aterro, parede da contenção e superfície de ruptura (esquerda); polígono de forças correspondente (direita), modificado de Kramer, 1996.

Figura 2.12 - Cunha passiva de solo delimitada pela superfície do aterro, parede da contenção e superfície de ruptura (esquerda); polígono de forças correspondente (direita).

Figura 3.1 - a) Forças atuando sobre a cunha ativa no método de

Mononobe-Okabe, b) polígono de forças incluindo as forças pseudo-estáticas $\mathrm{k}_{\mathrm{h}} \mathrm{W}$ e $\mathrm{k}_{\mathrm{v}} \mathrm{W}$ (modificado de Kramer, 1996).

Figura 3.2 - a) Forças atuando sobre a cunha passiva no método de Mononobe-Okabe, b) polígono de forças incluindo as forças pseudo-estáticas $\mathrm{k}_{\mathrm{h}} \mathrm{W}$ e $\mathrm{k}_{\mathrm{v}} \mathrm{W}$ (modificado de Kramer, 1996).

Figura 3.3 - Geometria da cortina e forças atuantes na cunha de solo (modificado de Kramer, 1996)

Figura 3.4 - Comparação da distribuição de tensões horizontais normalizadas na interface solo - estrutura obtidas pelos métodos M-O e Steedman-Zeng assumindo $k_{h}=0.2$ e H/ $\lambda=0.3$ (de Steedman e Zeng, 1990).

Figura 3.5 - Localização da posição da componente dinâmica do empuxo ativo no instante do momento máximo de tombamento para $\mathrm{k}_{\mathrm{h}}=0.2$ (de Steedman e Zeng, 1990).

Figura 3.6 - Geometria do problema investigado por Wood (1973).

Figura 3.7 - Fator de empuxo adimensional para várias geometrias L/H e valores do coeficiente de Poisson v (de Wood, 1973).

Figura 3.8 - Fator de momento adimensional para várias geometrias $\mathrm{L} / \mathrm{H}$ e valores do coeficiente de Poisson v. (de Wood, 1973).

Figura 3.9 - Processo de correção da linha base.

Figura 3.10 - Variação da razão de amortecimento critico normalizada em relação à frequência angular, (Itasca, 2005).

Figura 3.11 - Limite superior da curva de redução do módulo de cisalhamento para areias (Seed \& Idriss, 1970).

Figura 4.1 - Diagrama de corpo livre para uma estrutura de contenção (modificado de Richards, 1979). 
Figura 4.3 - Efeito da aceleração vertical sobre o fator de inércia da estrutura (modificado de Richards R., 1979).

Figura 4.4 - Efeito do ângulo de atrito na interface solo-estrutura sobre o fator de inércia da estrutura (modificado de Richards R., 1979).

Figura 4.5 - Variação com a aceleração horizontal do fator de empuxo de solo $F_{T}$, do fator de inércia da estrutura $F_{I}$ e do fator dinâmico combinado $F_{w}$. (modificado de Richards R., 1979).

Figura 4.6 - Esquema para cálculo dos deslocamentos permanentes da estrutura de contenção (modificado de Richards R., 1979).

Figura 4.7 - Histórias de aceleração e de velocidade para o solo e estrutura, considerando registro sísmico do terremoto El Centro 1940. Aceleração de corte 0,1g (modificado de Richards R., 1979).

Figura 4.8 - História dos deslocamentos permanentes da estrutura em relação ao solo, para o terremoto de El Centro - 1940 (modificado de Richards R., 1979).

Figura 4.9 - Deslocamento relativa máximo da estrutura com a variação da aceleração horizontal de corte $a_{h}=k_{h} g$, considerando terremoto de El Centro -1940 (modificado de Richards R., 1979).

Figura 4.10 - Curvas envoltórias de deslocamentos permanentes para os registros de aceleração investigados por Franklin e Chang -1977 (modificado de Richards R., 1979).

Figura 5.1 - Geometria do problema. Distâncias em metros.

Figura 5.2 - Malha de elementos finitos triangulares (6 nós) e condições de contorno.

Figura 5.3 - Determinação de tensões nos cantos de estruturas: a) sem elementos de interface; b) considerando elementos de interface (manual Plaxis v8.2)

Figura 5.4 - Fatores de segurança e posições das superfícies de ruptura determinadas.

Figura 5.6 - Variação dos coeficientes de empuxo ativo com a aceleração horizontal.

Figura 5.7 - Registros da aceleração do terremoto Mammoth Lake (1980) normalizados para aceleração máxima de $0,1 \mathrm{~g}$ e 0,3g. 
Figura 5.8 - Malha de elementos finitos, com contornos laterais silenciosos.

Figura 5.9 - Método das fatias de Morgenstern-Price para determinação

do fator de segurança pseudo-estático FS = 1 correspondendo à

aceleração horizontal de corte ou de escoamento $\mathrm{a}_{\mathrm{y}}$.

Figura 5.10 - Acelerações máximas na superfície do solo para sismo

Mammoth Lake com aceleração máxima normalizada de $0.1 \mathrm{~g} \mathrm{e} \mathrm{a}_{\mathrm{y}}=0.14 \mathrm{~g}$.

Figura 5.11 - Acelerações máximas na superfície do solo para sismo

Mammoth Lake com aceleração máxima normalizada de $0.3 \mathrm{~g}$ e $\mathrm{a}_{\mathrm{y}}=0.14 \mathrm{~g}$.

Figura 5.12 - Acelerações máximas na superfície do solo para sismo

Mammoth Lake com aceleração máxima normalizada de $0.3 \mathrm{~g}$ e $\mathrm{a}_{\mathrm{y}}=0.23 \mathrm{~g}$.

Figura 5.13 - Velocidades máximas calculadas na superfície do

solo considerando sismo Mammoth Lake normalizado com aceleração

horizontal máxima $0.1 \mathrm{~g}$.

Figura 5.14 - Velocidades máximas calculadas na superfície do solo

considerando sismo Mammoth Lake normalizado com aceleração horizontal máxima $0.3 \mathrm{~g}$.

Figura 5.15 - História dos deslocamentos do muro para sismo com aceleração máxima normalizada $0,1 \mathrm{~g}$.

Figura 5.16 - História dos deslocamentos do muro para sismo com aceleração máxima normalizada $0,3 \mathrm{~g}$.

Figura 5.17 - Histórias dos deslocamentos do muro com diferentes tipos de interfaces, aceleração de pico $0.1 \mathrm{~g}$ e $\xi=5 \%$.

Figura 5.18 - Histórias dos deslocamentos do muro com diferentes tipos de interfaces, aceleração de pico $0.1 \mathrm{~g}$ e $\xi=10 \%$.

Figura 5.19 - Histórias dos deslocamentos do muro com diferentes tipos de interfaces, aceleração de pico $0.3 \mathrm{~g}$ e $\xi=5 \%$.

Figura 5.20 - Histórias dos deslocamentos do muro com diferentes tipos de interfaces, aceleração de pico $0.3 \mathrm{~g}$ e $\xi=10 \%$.

Figura 5.21 - Histórias dos deslocamentos do topo e da base, aceleração de pico $0.3 \mathrm{~g}$ e $\mathrm{R}_{\text {inter }}=0.5$.

Figura 5.22 - Histórias dos deslocamentos do topo e da base, aceleração de pico $0.1 \mathrm{~g}, \mathrm{R}_{\text {inter }}=0.8$. 
Figura 5.23 - Histórias dos deslocamentos do topo e da base, aceleração de pico $0.3 \mathrm{~g}, \mathrm{R}_{\text {inter }}=0.8$.

Figura 5.24 - Variação no tempo do coeficiente de empuxo ativo para aceleração máxima normalizada do sismo igual a $0,1 \mathrm{~g}$ e $0.3 \mathrm{~g}$.

Figura 5.25- Variação com o tempo do ponto de aplicação do empuxo ativo para sismo com aceleração máxima normalizada $0,1 \mathrm{~g}$ e $0.3 \mathrm{~g}$.

Figura 5.26 - Malha deformada de elementos finitos no instante $\mathrm{t}=12 \mathrm{~s}$

considerando sismo com aceleração máxima normalizada de $0,3 \mathrm{~g}$ e $\xi=5 \% .102$ Figura A.1 - Principais componentes do modelo de bloco rígido deslizante (Hynes-Griffin e Franklin, 1984)

Figura A.2 - Procedimento da dupla integração no tempo no método de Newmark-Smith (1995). 


\section{Lista de tabelas}

Tabela 2.1 - Valores de $\mathrm{K}_{\mathrm{o}}$ (Lemos Machado S, 1997). 30

Tabela 2.2 - Métodos aproximados de análise de estruturas de contenção. 32

Tabela 2.3 - Valores típicos do ângulo de atrito na interface solo - estrutura

(NAVFAC, 1982)

Tabela 3.1 - Valores típicos da razão de amortecimento crítico (Richart et al., 1970).

Tabela 4.1 - Valores de médias e desvios padrão para análise de deslocamentos permanentes de muros de gravidade (Whitman e Liao, 1985).

Tabela 5.1 - Valores típicos de alguns fatores de redução de resistência $\mathrm{R}_{\text {inter }}$ (Pérez More, 2003).

Tabela 5.2 - Propriedades dos materiais usados na análise.

Tabela 5.3 - Resultados obtidos com o método pseudo-estático de MononobeOkabe e com a simulação numérica pelo método dos elementos finitos.

Tabela 5.4 - Valores de $\mathrm{c}_{1}$ e $\mathrm{c}_{2}$ em função do coeficiente de Poisson $v$

(White et al, 1977).

Tabela 5.4 - Acelerações máximas na superfície do terreno para sismos com aceleração máxima normalizada de $0.1 \mathrm{~g}$ e $0.3 \mathrm{~g}$.

Tabela 5.5 - Velocidades máximas na superfície do terreno para sismos com aceleração máxima normalizada de $0.1 \mathrm{~g}$ e $0.3 \mathrm{~g}$.

Tabela 5.6 - Deslocamentos permanentes para $0.1 \mathrm{~g}$ e $0.3 \mathrm{~g}$ calculados pelo método de Richards-Elms, para $\mathrm{a}_{\mathrm{y}}=0.14 \mathrm{~g}$.

Tabela 5.7 - Deslocamentos permanentes para $0.3 \mathrm{~g}$ calculados pelo método de Richards-Elms para $\mathrm{a}_{\mathrm{y}}=0.23 \mathrm{~g}$.

Tabela 5.8 - Deslocamentos permanentes para $0.1 \mathrm{~g}$ e $0.3 \mathrm{~g}$ calculados pelo método de Whitman-Liao para $\mathrm{a}_{\mathrm{y}}=0.14 \mathrm{~g}$

Tabela 5.9 - Deslocamentos permanentes para $0.1 \mathrm{~g}$ e $0.3 \mathrm{~g}$ calculados pelo método de Whitman-Liao para $\mathrm{a}_{\mathrm{y}}=0.23 \mathrm{~g}$.

Tabela 5.10 - Deslocamentos permanentes do muro de gravidade para sismos normalizados com aceleração máxima $0,1 \mathrm{~g}$ e $0,3 \mathrm{~g}$, PLAXIS. 


\section{Lista de Símbolos}

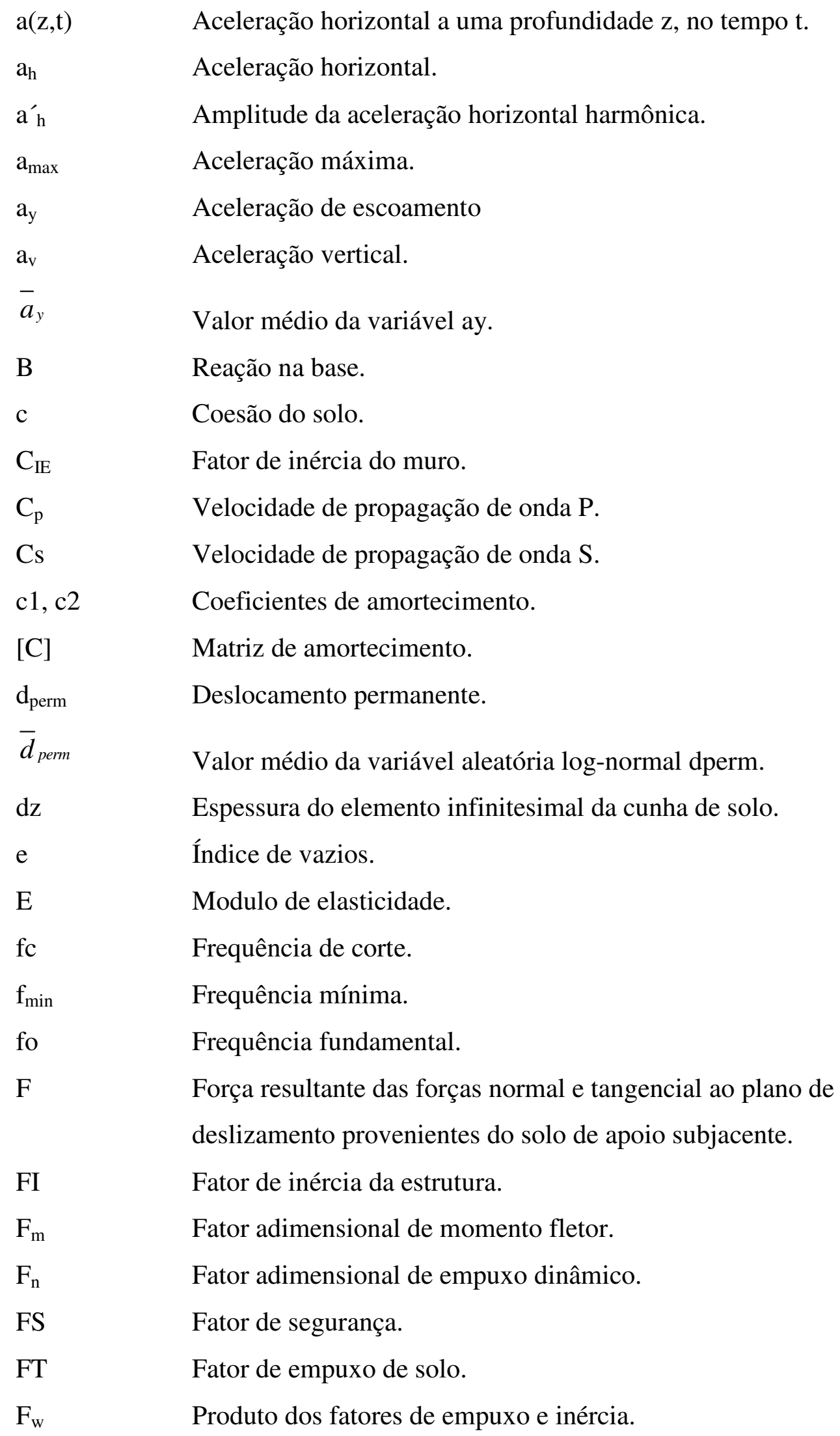


Aceleração da gravidade.

G

Módulo de cisalhamento.

h

Posição do ponto de aplicação da força resultante (empuxo ativo total).

$\mathrm{h}_{\mathrm{d}} \quad$ Ponto de aplicação do empuxo dinâmico.

$\mathrm{H}$

Altura da estrutura.

IP

Índice de plasticidade.

$\mathrm{k}_{\mathrm{h}}$

Coeficiente sísmico na direção horizontal.

$\mathrm{k}_{\mathrm{h}} *$

Coeficiente sísmico horizontal crítico.

$\mathrm{k}_{\mathrm{v}}$

Coeficiente sísmico na direção vertical.

$\mathrm{K}_{\mathrm{A}}$

Coeficiente de empuxo ativo.

$\mathrm{K}_{\mathrm{AE}}$

Coeficiente de empuxo ativo na condição pseudo-estática.

$\mathrm{K}_{\mathrm{o}}$

Coeficiente de empuxo no repouso.

$\mathrm{K}_{\mathrm{P}}$

Coeficiente de empuxo passivo.

$\mathrm{K}_{\mathrm{PE}}$

Coeficiente de empuxo passivo na condição pseudo-estática.

$[\mathrm{K}]$

Matriz de massa.

LL

Limite liquido.

LP

Limite plástico.

$\mathrm{m}(\mathrm{z})$

Massa do elemento infinitesimal da cunha de solo.

M

Variável aleatória com distribuição log-normal.

$\bar{M}$

Valor médio de uma variável aleatória M com distribuição log-

Normal.

[M]

Matriz de rigidez.

$\mathrm{N}$

Componente normal de B.

OCR

Razão de pré-adensamento do material.

$\mathrm{p}_{\mathrm{a}}$

Tensão horizontal ativa.

$\mathrm{p}_{\mathrm{P}}$

Tensão horizontal passiva.

$\mathrm{P}_{\mathrm{A}} \quad$ Empuxo ativo.

$\mathrm{P}_{\mathrm{AE}} \quad$ Empuxo ativo total.

$\mathrm{P}_{\mathrm{P}} \quad$ Empuxo passivo.

$\mathrm{P}_{\mathrm{PE}} \quad$ Empuxo passivo total.

Q Variável aleatória com distribuição log-normal.

$\mathrm{Q}_{\mathrm{h}}(\mathrm{t}) \quad$ Componente horizontal da força de inércia. 


\begin{tabular}{|c|c|}
\hline $\bar{Q}$ & Valor médio da variável aleatória log-normal Q. \\
\hline $\mathrm{R}_{\text {inter }}$ & Fator de redução de resistência da interface. \\
\hline SV & Onda de propagação vertical. \\
\hline $\mathrm{t}$ & Tempo. \\
\hline $\mathrm{T}$ & Componente tangencial de B. \\
\hline $\mathrm{U}$ & Sub-pressão. \\
\hline $\mathrm{V}_{\max }$ & Velocidade máxima. \\
\hline $\mathrm{W}$ & Peso da cunha de solo. \\
\hline $\mathrm{W}_{\mathrm{w}}$ & Peso do muro de contenção. \\
\hline $\mathrm{z}$ & Profundidade. \\
\hline$\alpha$ & Constante de amortecimento proporcional a massa. \\
\hline$\alpha_{\mathrm{A}}$ & Ângulo da superfície de ruptura em condições ativas. \\
\hline$\alpha_{\mathrm{AE}}$ & $\begin{array}{l}\text { Ângulo da superfície de ruptura com a horizontal, na condição } \\
\text { ativa e pseudo-estática. }\end{array}$ \\
\hline$\alpha_{\mathrm{P}}$ & Ângulo da superfície de ruptura em condições passivas. \\
\hline$\alpha_{\mathrm{PE}}$ & $\begin{array}{l}\text { Ângulo da superfície de ruptura com a horizontal, na condição } \\
\text { passiva e pseudo-estática. }\end{array}$ \\
\hline$\beta$ & Ângulo de inclinação do aterro. \\
\hline$\beta^{\prime}$ & Constante de amortecimento proporcional a rigidez. \\
\hline$\delta$ & Ângulo de atrito da interface solo - estrutura. \\
\hline$\Delta \mathrm{K}_{\mathrm{AE}}$ & Incremento dinâmico do coeficiente de empuxo. \\
\hline$\Delta \mathrm{M}_{\mathrm{eq}}$ & Momento de tombamento dinâmico. \\
\hline$\Delta \mathrm{P}_{\mathrm{eq}}$ & Empuxo ativo dinâmico. \\
\hline$\Delta \mathrm{P}_{\mathrm{AE}}$ & Componente pseudo-estática do empuxo ativo total. \\
\hline$\Delta \mathrm{P}_{\mathrm{PE}}$ & Componente pseudo-estática do empuxo passivo total. \\
\hline$\Delta \ell$ & Tamanho do elemento da modelagem. \\
\hline$\phi$ & Ângulo de atrito do solo. \\
\hline$\phi_{b}$ & Ângulo de atrito na interface do solo de fundação - estrutura. \\
\hline$\gamma$ & Peso específico do solo. \\
\hline$\lambda$ & Comprimento de onda SV. \\
\hline$\theta$ & Ângulo entre o solo e a face vertical do muro. \\
\hline$\rho$ & Massa específica do solo. \\
\hline
\end{tabular}




\begin{tabular}{|c|c|}
\hline$\sigma_{\text {ay }}$ & Desvio padrão de $\mathrm{a}_{\mathrm{y}}$. \\
\hline$\sigma_{\operatorname{lnM}}$ & Desvio padrão da variável M. \\
\hline$\sigma_{\text {lnd }}^{2}$ & Variância da variável d. \\
\hline$\sigma_{\operatorname{lnQ}}$ & Desvio padrão da variável Q. \\
\hline$\sigma_{\mathrm{n}}$ & Tensão normal aplicada. \\
\hline$\sigma_{\phi}$ & Desvio padrão do ângulo $\phi$. \\
\hline$\sigma_{\delta}$ & Desvio padrão do ângulo $\delta$. \\
\hline$\sigma_{\mathrm{s}}$ & Tensão cisalhante aplicada. \\
\hline$\sigma_{x}^{\prime}$ & Tensão horizontal. \\
\hline$\sigma_{x p}^{\prime}$ & Tensão horizontal passiva. \\
\hline$\sigma_{x o}^{\prime}$ & Tensão horizontal inicial. \\
\hline$\sigma_{\text {xa }}^{\prime}$ & Tensão horizontal ativa. \\
\hline$\sigma^{\prime}{ }_{v}$ & Tensão vertical. \\
\hline$\tau$ & Tensão cisalhante. \\
\hline$v$ & Coeficiente de Poisson. \\
\hline$v_{\mathrm{n}}$ & Velocidade normal da particular. \\
\hline$v_{\mathrm{s}}$ & Velocidade tangencial da particular. \\
\hline$v_{s}^{\prime}$ & $\begin{array}{l}\text { Velocidade de propagação da onda cisalhante vertical no maciço } \\
\text { de solo. }\end{array}$ \\
\hline$\omega$ & Frequência angular de vibração. \\
\hline$\xi$ & Razão de amortecimento. \\
\hline$\sum H$ & Somatório das forças atuantes na direção horizontal. \\
\hline$\sum V$ & Somatório das forças atuantes na direção vertical. \\
\hline$\Psi^{\prime}$ & Ângulo de dilatância do solo. \\
\hline
\end{tabular}

